hep-th/0203016

DFAQ-02/TH/01

ITEP-TH-10/02

LPTHE-02-15

IHES/P/02/12

LPT-Orsay-02/13

OUTP-02-05P

\title{
On the Deconstruction of Time
}

\author{
Z. Berezhiani ${ }^{a, b}$, A. Gorsky ${ }^{c, d}$ and I.I. Kogan ${ }^{c, e, f, g}$ \\ ${ }^{a}$ Dipartimento di Fisica, Università di L'Aquila, I-67010 Coppito AQ, and \\ INFN, Laboratori Nazionali del Gran Sasso, I-67010 Assergi AQ, Italy \\ ${ }^{b}$ Andronikashvili Institute of Physics, 380077 Tbilisi, Georgia \\ ${ }^{c}$ Institute of Theoretical and Experimental Physics, \\ B.Cheremushkinskaya 25, 117259 Moscow, Russia \\ ${ }^{d}$ LPTHE, Université Paris VI, 4 Place Jussieu, Paris, France \\ e Theoretical Physics, Department of Physics, Oxford University \\ 1 Keble Road, Oxford, OX1 3NP, UK \\ ${ }^{f}$ IHES, 35 route de Chartres, 91440 Bures-sur-Yvette, France \\ $g$ Laboratoire de Physique Théorique, Université de Paris XI, \\ 91405 Orsay Cédex, France
}

\begin{abstract}
In this note we discuss the possibility to get a time rather than space in the scenario of (de)construction of new dimension.
\end{abstract}

1. Recently it was suggested in refs. [1, 2] that a four-dimensional gauge theory with a large gauge symmetry behaves in the infrared region in a manner which is very similar to a five-dimensional gauge theory with a smaller gauge group. This leads to an idea of (De)construction of extra dimensions, i.e that extra dimensions do not exist at fundamental level and emerge dynamically in the infrared limit. The basic idea of (De)construction is the following [1, 2]. One starts from a theory with a chain of gauge symmetries $G_{1} \times G_{2} \times \ldots \times G_{N}$ where all groups $G_{i}$ are identical, i.e. we have 
$N$ copies of the same gauge group $G$. Matter is represented by a set of scalar fields $\Phi_{i, i+1}$ 's each of which is transformed as a fundamental representation with respect to symmetry $G_{i}$ and anti-fundamental with respect to the neighbor $G_{i+1}$. These scalar fields $\Phi_{i, i+1}$ develop non-zero VEVs and hence the total gauge symmetry will be broken down to a diagonal subgroup G. For simplicity, let us consider the case when $G=U(1)$, i.e. scalars $\Phi_{i, i+1}$ have charges $Q_{i}=1$ and $Q_{i+1}=-1$ with respect to the neighbor groups $U(1)_{i}$ and $U(1)_{i+1}$. The system is described by the Lagrangian

$$
\mathcal{L}=-\frac{1}{4 g^{2}} \sum_{i=1}^{N} F_{(i) \mu \nu} F_{(i)}^{\mu \nu}-\sum_{i=1}^{N} D_{\mu} \Phi_{i, i+1}^{\dagger} D^{\mu} \Phi_{i, i+1}
$$

(the signature $(-,+,+,+)$ is chosen), where the covariant derivative is defined as $D_{\mu}=\partial_{\mu}+\mathrm{i} \sum_{i} A_{(i) \mu} T^{(i)}$ and $T^{(i)}$ are the generators of the gauge symmetry with respect to the group number $i$. Therefore, for a field $\Phi_{i, i+1}$ one gets

$$
D_{\mu} \Phi_{i, i+1}=\partial_{\mu} \Phi_{i, i+1}+\mathrm{i}\left(A_{(i) \mu}-A_{(i+1) \mu}\right) \Phi_{i, i+1} .
$$

When order parameter $\Phi_{i, i+1}$ acquires non-zero VEV one has

$$
\Phi_{i, i+1}=v \exp \left(\mathrm{i} \phi_{i, i+1} / \sqrt{2} g v\right) .
$$

Neglecting in the IR limit the kinetic energy $\partial_{\mu} v \partial^{\mu} v$, one can see that the scalar contribution to the Lagrangian equals to

$$
\frac{1}{2 g^{2}} \sum_{i=1}^{N}\left(\partial_{\mu} \phi_{i, i+1}-\sqrt{2} g v\left(A_{(i+1) \mu}-A_{(i) \mu}\right)\right)\left(\partial^{\mu} \phi_{i, i+1}-\sqrt{2} g v\left(A_{(i+1)}^{\mu}-A_{(i)}^{\mu}\right)\right) .
$$

This term has the structure of a discrete version of $F_{\mu, 5} F^{\mu, 5}$ where phase $\phi_{i, i+1}$ is a phase for link variable for component $A_{5}(x, i)$ and

$$
\sqrt{2} g v\left(A_{(i+1) \mu}-A_{(i) \mu}\right) \rightarrow \partial_{5} A_{\mu}(x, i)+O\left(v^{-1}\right) \partial_{5}^{2} A_{\mu}(x, i) .
$$

The lattice spacing $a$ is related to the condensate $v$ through the relation

$$
\sqrt{2} g v a=1
$$

so continuous limit corresponds to large $v$. In this model one gets a "transverse lattice" description of a full $4+1$ gauge theory where the size of extra space $L$ is proportional to the number of independent gauge symmetries in an unbroken phase:

$$
L=N a=\frac{N}{\sqrt{2} g v} .
$$

It was shown recently that the deconstruction survives nonperturbatively in the supersymmetric case [ [3] and two compact dimensions instead of one can be constructed along this way if the full lattice of nonperturbative states in taken into account [ [ब]. Moreover, recently there have been attempts of introducing gravity in this scenario [5]. On other phenomenological applications of this approach see [6].

\footnotetext{
${ }^{1}$ Alternatively, instead of fundamental scalar fields one can consider some bilinear fermion condensates [1], but this is not so important.
} 
2. Of course this construction can be repeated for space-time with any dimensions and starting from $d+1$-dimensional space-time we can get $(d+1)+1$ dimensional space-time in the infrared limit. One can immediately ask the following question: is it possible to start from $d$-dimensional space and get $d+1$ dimensional space-time? In other words, can we get time out of nothing using (De)constructing ?

The answer on this question appears to be positive, but we have to work with the system involving the apparently tachyonic degrees of freedom. Actually the different dependence of the vector and scalar degrees of freedom on the metric seems to be crucial for our purpose. Therefore, we explicitly restore the dependence on the metric in gauge action:

$$
\mathcal{L}=-\frac{1}{4 g^{2}} \sum_{i=1}^{N} g^{m n} g^{k l} F_{(i) m k} F_{(i) n l}-\sum_{i=1}^{N} g^{m n} D_{m} \Phi_{i, i+1}^{\dagger} D_{n} \Phi_{i, i+1}
$$

and carefully study the metric which arises after deconstruction.

Consider first a space-time with the metric $g_{n m}=\operatorname{diag}(-++\ldots+)$, in the spirit of the example discussed in refs. [1,2]. For simplicity, let us consider the case $d=3$ with $g_{n m}=\operatorname{diag}(-++)$. Lagrangian (8) leads to a well-defined action

$$
S=\int d^{3} x \sqrt{-g} \mathcal{L}
$$

For the choice of metric $g_{n m}=\operatorname{diag}(+--)$, we have the wrong sign in front of the scalar part of the action (it would be ghost-like), but the gauge part is all right. Transition from signature $(-++)$ to the signature $(+--)$ is nothing but transformation

$$
g_{n m} \rightarrow-g_{n m}
$$

and obviously the Lagrangian of vector fields is invariant under this transformation, while the kinetic part of the scalar Lagrangian is not. Let us note that the path integral for the theory with the action (8) is defined with an oscillating exponent

$$
\int D A_{m}(x) \prod_{i} D \Phi_{i, i+1}(x) \exp \left\{-\mathrm{i} \int d^{3} x \sqrt{-g} \mathcal{L}\right\}
$$

In the low energy limit this path integral describes a gauge (Maxwell or Yang-Mills) theory including a matter in a $3+1$ dimensional space-time with one extra compact spatial direction and metric $G_{\mu \nu}=\operatorname{diag}(-+++)$.

Let us turn now now to our main observation and show how the time coordinate can be generated if the metric different from $g_{n m}=\operatorname{diag}(-++)$ is chosen before deconstruction. The most interesting possibility is to assume that our metric describes the Euclidean space, i.e. all directions have the same signature. There are formally two possibilities:

- To take metric $g_{E}=\operatorname{diag}(+++)$, i.e. all coordinates are like spatial coordinates in our original $2+1$ space-time. (Of course there is no time now - it exists only when we have quadratic form with indefinite sign.)

- To take metric $g_{L}=\operatorname{diag}(---)$, i.e. in our original $2+1$ 'space-time' all coordinates are like temporal coordinates, or formally they are the spatial ones but the "kinetic" terms of $\Phi$-fields in (8) have the wrong sign. 
In the first case one can see that starting from $g_{E}$, after deconstruction one gets extra spatial coordinate and hence an Euclidean gauge theory in $d=4$ space:

$$
G_{E}^{M N}=\delta^{M N}
$$

with the low-energy action

$$
-\mathrm{i} S=-\mathrm{i} \frac{1}{4 g^{2}} \int d^{3} x d y \sqrt{G_{E}} F_{M N} F_{M N}
$$

where the extra ' $\mathrm{i}$ ' amounts from the factor $\sqrt{-g}$ and it cancels with the factor ' $\mathrm{i}$ ' in the path integral (11), thus yielding an Euclidean field theory with a real path integral:

$$
\int D A_{M}(x, y) \exp \{-S[A]\}
$$

Let us consider now the second choice, i.e. when we have metric $g_{L}=\operatorname{diag}(---)$. It is easy to see that in this case relative signs of gauge and scalar sectors are different. If one considers a lattice regularization for the space one can see that scalar part corresponds to antiferromagnetic coupling between nearest neighbors, contrary to the ferromagnetic coupling in the space case: the state where neighbor fields are close to each other does not not correspond to minimum but to maximum implying the existence of the unstable mode. One can easily see that repeating the same steps we do not change anything in a gauge part (which becomes now the magnetic field part of the action) but because of the change of the sign in front of scalar part we effectively get an electric field contribution.

The naive way to get the electric field contribution $F_{0 m} F^{0 m}=-F_{0 m} F_{0 m}$ out of the Goldstone part is just to identify $\phi_{i, i+1}(x)$ as a scalar potential $A_{0}(x, i)$ and approximate in the expression

$$
F_{0 m}(x, i)=\partial_{m} \phi_{i, i+1}(x)-\sqrt{2} g v\left(A_{(i+1) m}(x)-A_{(i) m}(x)\right)
$$

the combination $A_{(i+1) m}(x)-A_{(i) m}(x)$ as a time derivative $\frac{d A_{m}}{d t} \delta t$. In this view the step of the "time lattice" is nothing but the inverse value of the condensate, $\delta t=1 / \mathrm{gv}$. The higher derivative terms are suppressed by the large value of the condensate.

As a result, we get into the theory in a space-time having the Minkowski metric

$$
G_{L}^{\mu \nu}=\operatorname{diag}(-,+,+,+) .
$$

Since the factor $\sqrt{-G}$ should appear in this case there is no extra 'i' when we go from 3 to $3+1$ dimensions. Therefore, we derive the correct path integral (with complex phase) for the gauge theory in Lorentzian space-time. Note also that the deconstruction effectively restores the symmetry $G_{M N} \rightarrow-G_{M N}$ which becomes the effective low energy symmetry.

One can be more precise and look more carefully on the "mass term" for the "W-bosons", which can be easily found from the action. In refs. [1, 2] this term was immediately identified with KK masses using the mode expansion of the gauge field $A\left(t, \vec{x}, x_{5}\right)$. Now, since we are hunting for the time, it is natural to assume that such term amounts from the mode expansion of $A_{m}(t, \vec{x})$ in $t$ variable. As far as the eigenvalues of the mass matrix have the structure $m_{k}=g v \sin (k / N)$ at large $\mathrm{N}$ the 
linear spectrum of frequencies $\omega_{k} \propto k$ has to be somehow explained. In the KK case it is just the consequence of the periodic or $Z_{2}$ orbifold boundary conditions. However in the case of time periodic conditions in the Minkowski space are not acceptable. Hence, in our case we could have only a kind of orbifold boundary conditions on the "boundary of the Universe" or free boundary conditions and infinite N. In principle one can consider finite $N$ and this case will correspond to the "Universe" which originates at some moment and which existence will be terminated at some later moment. Perhaps this approach could be also useful to discuss the periodic time, for example the case of $A d S$ space-time.

Since the time direction emerges dynamically we have to examine the Gauss law selecting the gauge invariant states at the quantum level. Let us compare how Gauss law and gauge invariance are realized in the KK and "time" cases. In first case we have

$$
\begin{aligned}
\partial_{m} E_{(i) m} & =\rho_{i} \\
-\rho_{i} & =\partial_{0} \phi_{i, i+1}(x)-\sqrt{2} g v\left(A_{(i+1) 0}(x)-A_{(i) 0}(x)\right) \\
& -\partial_{0} \phi_{i-1, i}(x)-\sqrt{2} g v\left(A_{(i) 0}(x)-A_{(i-1) 0}(x)\right) .
\end{aligned}
$$

One can easily see that in a continuum limit the density $\rho_{i}$ becomes nothing but $-\partial_{5} E_{5}$. As a result we get a five dimensional Gauss law

$$
\partial_{m} E_{m}+\partial_{5} E_{5}=0
$$

The totally different story appears when we want to get time out of deconstruction. In this case we do not have electric field to start with. One can see that the Gauss law $\partial_{m} E_{m}=0$ which is supposed to be valid at any time moment $i$ can be written as

$$
\partial_{m} F_{0 m}(x, i)=\partial^{2} \phi_{i, i+1}(x)-\sqrt{2} g v\left(\partial_{m} A_{m}(x, i+1)-\partial_{m} A_{m}(x, i)\right)=0
$$

One can choose all $\phi_{i, i+1}(x)=0$ (this is $A_{0}=0$ gauge). The Gauss law in this case reads as

$$
\partial_{m} A_{m}(x, i+1)=\partial_{m} A_{m}(x, i) .
$$

and corresponds to the time independent gauge fixing.

Let us also comment on the possible relation between the quasiclassical nonperturbative configurations. It was argued in KK supersymmetric case that nonperturbative configurations are mapped to each other under deconstruction [3]. In the 'time' case we would like to get, for instance, instanton in the deconstructed theory. It can be obtained indeed, considering the infinite arrow of monopoles in $\mathrm{d}=3$ theory along the "time" direction.

3. Now let us briefly mention what are the physical consequences of the picture proposed. First note that the perception of time as a chain of the ordered events with no return to the past is just the fact that we are measuring observables in the $i$-th sector only once. After we measured it we have to measure the next one - and never can return back - because the wave function in that sector is already defined. Second, it is natural to ask if a kind of appearance (or disappearance) of the time dimension in the IR(UV) limits similar to what happens in the KK case is possible. In the KK case the condensate can be destroyed at large energies, 
yielding the effective disappearance of the fifth dimension. One can relate this with the uncertainty relation $\Delta x_{5} \Delta p_{5} \geq 1$ - when we try to localize the position in space we bring such an uncertainty in the momentum that causes the destruction of the condensate. If for example we shall destroy the condensate on the link $(i, i+1)$ it will cause the creation of two disconnected worlds. In the "time" case one could also imagine the "dynamical" disappearance of the condensate which would look like that "time disappears". Again, if we try to destroy a condensate just on one link, it will cause also emergence of two disconnected worlds - but now they will be disconnected in time. Now we have the uncertainty relation $\Delta E \Delta t \geq 1$, and because we have the time quanta $\Delta t \sim v^{-1}$, one can not have $\Delta E \leq v$.

Our perception of time is a causally ordered sequence of the processes of measurement each of which can happen only once. If time emerges in the way as we have just described, this would mean that the observer can make a measurement for all gauge fields - but only once. In some sense the full evolution is just one complete measurement. This sounds quite natural indeed. When one is asking question about what happens when we repeat measurement it is based on the assumption that we can measure something again later. But later means later in time - and if time itself emerges dynamically this question simply can not be asked. There is only one measurement for each sector - which means that you can not return back in the past and reobserve the things.

Among other interesting points to be questioned is the deconstruction of $d=2 \mathrm{YM}$ theory. Since in $\mathrm{d}=2$ the $\mathrm{YM}$ theory is topological, only zero modes on the cylinder are relevant and all higher KK modes can be cut off safely. The theory which amounts to d=2 YM theory after deconstruction can be presented as $N$ copies of quantum mechanics where $N$ defines the radius of the cylinder. However, since only zero mode works, only one copy of quantum mechanics is relevant. Now turn to the question concerning the signature of $d=2$ theory. Before deconstruction, the issue of signature in quantum mechanics is subtle since we have to deal with "world-line" (time) and "target" (coordinate) simultaneously. It can be well defined only for the relativistic particle since the length of the world-line is defined with some metric.

On the other hand $\mathrm{d}=2 \mathrm{YM}$ theory at large $\mathrm{N}$ is equivalent to $\mathrm{c}=1$ string theory, that is what we are talking about is the deconstruction of $c=1$ string theory from the set of copies of quantum mechanics. Moreover, from the viewpoint of $\mathrm{c}=1$ string we are deconstructing the target manifold since $\mathrm{d}=2 \mathrm{YM}$ theory is defined on the target from the stringy point of view. The way how the second dimension emerges in $\mathrm{c}=1$ string is known - it is the Liouville mode while the $\mathrm{c}=1$ string theory can be defined via matrix quantum mechanics indeed. Hence the issue of the resulting metric in $\mathrm{d}=2$ theory is related with the sign in front of the Liouville contribution to the action. Usually it is assumed that Liouville field plays the role of time. One more potential question concerns the deconstruction of $(0+1)$ theory (quantum mechanics) from the copies of $(0+0)$ (matrix model). This has something to do with the M(atrix) model deconstruction of D0 brane from D-instantons.

It is known how the deconstruction procedure can be formulated in terms of branes. For instance, to get the quiver models one could take the set of D3 branes on orbifolds in SUSY case [7] then $W$ bosons are represented by the strings connecting the pairs of D3 branes. When this paper was almost completed the preprint [8] appeared where new branes localized in time direction have been found. These branes are very 
natural objects to start with to get the new time like-coordinates in terms of the brane array leading to the group products. Since fundamental strings can end on them the spectrum of "masses" could be reproduced in a way similar to the KK case.

4. In conclusion let us make our main statement again. Starting from the action (8), it is possible to get a quantum field theory in a space-time where either extra spatial coordinate or extra time emerges via deconstruction. Alternatively one can get statistical field theory.

\section{Acknowledgments}

The work of Z.B. is partially supported by the MURST research grant "Astroparticle Physics", the work A.G. is supported in part by grants INTAS-00-00334 and CRDF-RP2-2247, and that of I.I.K. is supported in part by PPARC rolling grant PPA/G/O/1998/00567 and EC TMR grant HPRN-CT-2000-00152.

\section{References}

[1] N. Arkani-Hamed, A.G. Cohen and H. Georgi, Phys. Rev. Lett. 86 (2001) 4757 arXiv:hep-th/0104005

[2] C.T. Hill, S. Pokorski and J. Wang, Phys. Rev. D 64 (2001) 105005 arXiv:hepth/0104035.

[3] C. Csaki, J. Erlich, V.V. Khoze, E. Poppitz, Y. Shadmi and Y. Shirman, arXiv:hep-th/0110188.

[4] N. Arkani-Hamed, A.G. Cohen, D.B. Kaplan, A. Karch and L. Motl, arXiv:hepth/0110146.

[5] A. Sugamoto, arXiv:hep-th/0104241

M. Alishahiha, Phys. Lett. B 517 (2001) 406 arXiv:hep-th/0105153

M. Bander, Phys. Rev. D 64 (2001) 105021 arXiv:hep-th/0107130.

[6] H.C. Cheng, C.T. Hill, S. Pokorski and J. Wang, Phys. Rev. D 64 (2001) 065007

N. Arkani-Hamed, A.G. Cohen and H. Georgi, Phys. Lett. B 513 (2001) 232 arXiv:hep-ph/0105239.

C. Csaki, G. D. Kribs and J. Terning, Phys. Rev. D 65, 015004 (2002) arXiv:hepph/0107266.

K. Sfetsos, Nucl. Phys. B 612, 191 (2001) arXiv:hep-th/0106126.

W. Skiba and D. Smith, arXiv:hep-ph/0201056.

N. Arkani-Hamed, A. G. Cohen and H. Georgi, arXiv:hep-th/0109082.

T. Kobayashi, N. Maru and K. Yoshioka, arXiv: hep-ph/0110117

[7] M. R. Douglas and G. W. Moore, arXiv:hep-th/9603167.

[8] M. Gutperle and A. Strominger "Spacelike Branes", (arXiv:hep-th/0202210) 